\title{
VALORACIÓN DE LA EFICACIA PERCIBIDA DE LA DINÁMICA GRUPAL "¿QUÉ-TAL?" PARA EL AUTOCUIDADO Y APRENDIZAJE MUTUO EN UN EQUIPO ASISTENCIAL
}

\author{
EVALUATION OF THE THE DYNAMIC GROUP “WHAT'S UP?" PERCEIVED \\ EFFICACY FOR SELF-CARE AND MUTUAL LEARNING IN A HEALTH CARE TEAM
}

Javier Barbero', Patricia Fernández-Herreruela', Helena García-Llana², Olga MayoralPulido' y Víctor Jiménez-Yuste ${ }^{l}$

I Servicio Hematología y Hemoterapia Hospital Universitario La Paz

2 Servicio Nefrología Hospital Universitario La Paz-IdiPAZ

Resumen

El equipo de psicólogos del Servicio de Hematología del Hospital Universitario La Paz, viene realizando una dinámica semanal, como herramienta de autocuidado y aprendizaje mutuo. La finalidad es compartir la valoración de la semana en el ámbito personal y profesional, de manera formal y sistemática. A esta dinámica grupal de enfoque interdisciplinar la denominamos "¿Qué-tal?".

Objetivo: presentar la eficacia percibida de la dinámica por parte de los participantes de los últimos 6 años.

Método: estudio retrospectivo con una única medida en forma de cuestionario on-line semiestructurado, elaborado ad hoc. La muestra final consta de 41 participantes.

Resultados: Se evidencia la alta eficacia percibida de esta herramienta con una media de 8 sobre $10(D T=0,62)$. Las correlaciones más robustas resultaron ser la gestión emocional, apoyo mutuo, pertenencia grupal y la ayuda en el ámbito personal, $(p<0,01)$.

Conclusiones: Los mayores beneficios percibidos se refieren al ámbito emocional y a la relación grupal, seguidos del aprendizaje de habilidades profesionales. Se considera beneficiosa la implantación de programas preventivos de autocuidado para profesionales en contacto con intensa experiencia de sufrimiento.
Abstract

The team of psychologists in Hematology Service of La Paz University Hospital, has incorporated a weekly dynamic, as a tool of self-care and mutual learning. The purpose is to share the assessment of the week in the personal and professional areas, in a formal and systematic proceeding. We call it "What's-up?"

Objective: To present the perceived effectiveness of the dynamic by those who participated in it during the last 6 years.

Method: Retrospective study with a single measure in the form of semi-structured online questionnaire, developed ad-hoc. The final sample consists of $\mathbf{4 1}$ participants.

Results: High efficiency is perceived by these professionals of this dynamic, with an average of 8 over 10 (DS=0.62). The most robust correlations turned out to be the emotional management, mutual support, group belonging and aid in the personal sphere, all of them $(\mathbf{p}<0,01)$.

Conclusions: The greatest perceived benefits were related to the emotional level and the group relationship, followed by learning professional skills. The implementation of selfcare preventive programs for professionals in contact with intense experience of suffering is considered to be beneficial. 
Palabras clave: eficacia percibida, trabajo en equipo, autocuidado, dinámica grupal, sufrimiento.
Key words: Perceived-efficacy, teamwork, self-care, group dynamics, suffering.

\section{INTRODUCCIÓN}

Los que trabajamos en hospitales y muy especialmente, los que nos acercamos a la esfera emocional de las personas que sufren, estamos expuestos a una gran sobrecarga emocional. Zapf ${ }^{(1)}$ alude al término "trabajo emocional" como un aspecto importante dentro de los estresores laborales presentes en el ámbito hospitalario. Este fenómeno consiste en el esfuerzo realizado al intentar sincronizar tres variables: el evento impactante que se da en la situación laboral, la expresión emocional esperada por la organización y la experiencia emocional propia.

Figley ${ }^{(2)}$ recomienda a los que trabajan procurando el alivio del sufrimiento ajeno que desarrollen prácticas de autocuidado para prevenir el desgaste laboral.

La literatura nos habla de diversas propuestas de autocuidado como la revisión de casos clínicos ${ }^{(3)}$, el uso del sentido del humor en el contexto laboral ${ }^{(4)}$, el propio diálogo con los colegas sobre experiencias personales en el trabajo ${ }^{(5)}$, o la participación en actividades recreativas con los compañeros $^{(6)}$.

Actualmente despuntan estrategias más formalizadas de autocuidado en equipos asistenciales, como los grupos Debriefing, que pretenden facilitar la ventilación de los sentimientos y emociones relacionados con la experiencia traumática vivida. El propósito es reordenarla cognitivamente de forma adaptativa. Esta estrategia consta de 4 fases: 1. Introducción, donde el psicólogo que guía la dinámica se presenta especificando los objetivos de la intervención. 2. Narración, en esta fase los participantes comentan los hechos y sus reacciones personales e ideas acerca de lo ocurrido. 3. Reacción, en la que se promueve la liberación de las emociones vividas en dicha situación. 4. Educación, se informa de los síntomas comunes del trastorno de estrés postraumático, se entrega material informativo sobre estrategias de afrontamiento, etc. ${ }^{(7)}$

Otra dinámica que va extendiéndose tímidamente en el ambiente hospitalario es la Ilamada Grupo Balint. En ella participan diversos profesionales de la salud y un profesional de la salud mental (en los orígenes era un psicoanalista). Se presentan casos de la práctica médica y se comentan los aspectos psicológicos y relacionales. El papel del facilitador es invitar a estos profesionales a hablar sobre sus dificultades en la relación con los pacientes, los familiares y con el resto de compañeros del equipo. Se puede apreciar cómo en estas dinámicas se pretende fomentar la expresión emocional de los profesionales que acompañan la experiencia de sufrimiento ${ }^{(8)}$.

Parece importante normalizar las reacciones psicológicas de cara a fomentar un adecuado proceso de adaptación en entornos de frecuente sufrimiento. Como nos recuerda Viktor Frankl: "ante una situación anormal, como lo es acompañar el sufrimiento ajeno, la reacción anormal constituye una respuesta normal"(9).

El beneficio encontrado en la expresión emocional también ha sido descrito desde el marco de la psicología narrativa. Lakoff et al. ${ }^{(10)}$ nos identifica como sujetos narradores cuyos pensamientos que son esencialmente metafóricos, son manipulados en busca del significado. Accedemos a la realidad, como conjunto de problemas apenas estructurados a través de narrati$v s^{(10)}$. Un ejemplo de ello es la técnica de la escritura emocional de Pennebaker ${ }^{(11)}$, que ha demostrado cómo la expresión de 
las propias emociones puede prevenir a largo plazo problemas de salud, generando un aumento del sistema inmunológico. Esta autorrevelación emocional favorece el procesamiento del evento traumático, logrando un mejor ajuste físico y psicológico. Un ajuste, que en los que trabajamos con el sufrimiento humano resulta especialmente necesario compartir.

De ese modo, como dice Ramón Bayés ${ }^{(12)}$ en su abordaje del sufrimiento, cuando una persona experimenta una situación amenazante para su integridad, bien sea física o biográfica, evaluará los recursos de que cree disponer. Si se siente impotente para hacerle frente, si se encuentra indefenso, esta situación generará sufrimiento. Las estrategias grupales de autocuidado podrían centrarse en acortar la distancia entre la amenaza percibida y los recursos disponibles para afrontarla.

Otro modelo que nos es útil es el de la psicoterapia existencial de Yalom ${ }^{(13)}$. Es un enfoque terapéutico dinámico que se centra en problemáticas que tienen sus raíces en la existencia. Parte del supuesto de que la desesperación del paciente se debe a una incapacidad para desarrollar y mantener relaciones interpersonales gratificantes. Se considera que el paciente sufre la desesperación como resultado de una confrontación con su propia condición humana, con sus propias condiciones existenciales ${ }^{(13)}$. Nos recuerda que hay variables no tangibles, trascendentes, donde el límite de la vida de los otros inevitablemente nos afecta. Es importante disponer de un espacio para abordarlo de manera grupal.

El equipo de psicólogos del Servicio de Hematología del Hospital Universitario La $P a z$, viene realizando desde hace varios años, una dinámica sistemática semanal de autocuidado. La realización de este estudio pretende conocer los beneficios percibidos por sus usuarios a lo largo de los 6 últimos años.

\section{MATERIAL Y MÉTODOS}

Se realiza un estudio retrospectivo con una única medida en forma de cuestionario on line elaborado ad hoc.

\section{Participantes}

Se consideró para la muestra total a 50 profesionales que han rotado en el equipo de psicólogos del servicio de Hematología del Hospital Universitario La Paz en los últimos 6 años. Se contactó con ellos a través de una plataforma on line. Se obtuvieron 41 cuestionarios completos (tase de respuesta del $82 \%$ ) que forman la muestra final.

\section{Criterios de inclusión}

Mayores de 18 años que hayan rotado en el Servicio un mínimo de dos semanas y que accedan a participar de manera voluntaria y anónima.

\section{Proceso de elaboración del cuestionario}

Para la elaboración del instrumento se llevó a cabo una revisión de la literatura centrada en la fundamentación teórica de Pennebaker (1998), de la Autorrevelación Emocional, que explica cómo la expresión de las emociones en el recuerdo de hechos traumáticos vivenciados, logra mejorar el ajuste físico y psicológico.

Los miembros que componen el equipo actual de psicólogos de Hematología diseñaron un primer borrador del cuestionario. $\mathrm{Al}$ cabo de tres meses realizaron una evaluación sistemática sobe la comprensión, relevancia y no duplicidad de contenidos de los ítems inicialmente formulados. Si el acuerdo interjueces era igual o mayor del $80 \%$, respecto a la comprensión y la relevancia del ítem, el ítem se mantenía. Si la proporción de acuerdo en el mantenimiento del ítem era inferior al 50\% el ítem 
se eliminaba. Ningún ítem tuvo que ser eliminado. A la hora de efectuar la revisión y redacción final de los ítems se tuvieron presentes las observaciones y sugerencias concretas de los jueces.

\section{Cuestionario ad hoc $(\alpha=0,85)$}

El cuestionario consta de tres partes: datos sociodemográficos constituido por 8 preguntas; experiencia personal con la dinámica con 8 preguntas abiertas y valoración de las variables de la herramienta en formato likert de 0 a 10 . Ver anexo 1.

\section{Dinámica del “QQué-Tal?”}

Esta dinámica, consiste en una reunión semanal de equipo de una hora, en la que el coordinador modera las intervenciones. En este espacio cada miembro expone su experiencia en base a dos preguntas facilitadoras:

1. ¿Qué tal te ha ido la semana?

2. ¿Qué destacarías con respecto a pacientes, familiares o equipo?

Cada intervención finaliza con un feedback grupal no estructurado, constructivo hacia el participante que ha intervenido. En sí el "¿Qué-tal?" pretende crear un ambiente seguro y de escucha activa donde las intervenciones suelen ser breves.

\section{Objetivos del “¿Qué-tal?"}

a) Proveer de manera estable una dinámica explícita de autocuidado.

b) Reservar un tiempo explícito en la dinámica grupal para evitar que lo urgente se coma siempre los tiempos y las energías de lo importante. (No olvidemos que la presión asistencial puede fagocitar cualquier intento de autocuidado grupal).

c) Apoyar al equipo y sus profesionales en la dinámica de gestión del mundo emocional. d) Convertir al equipo asistencial en un potencial grupo de apoyo mutuo.

e) Prevención de burnout entre sus integrantes.

f) Disponer de un "termómetro" real de la temperatura del equipo y de las personas que lo integran en relación a la tarea y a la dinámica interna.

\section{Procedimiento}

Se elaboró una plataforma on line en donde se pudiesen volcar las respuestas de los participantes. Se alcanzó una muestra de 50 contactos con sus correspondientes e-mails. Se realizó el envío de un correo por parte del coordinador del equipo. En el correo se da la información sobre el estudio, explicitando que la respuesta expresa el consentimiento a participar en el mismo. Se establece un plazo de 15 días para cumplimentar de forma anónima el cuestionario a través de la plataforma. Transcurrido ese plazo se procedió a un envío recordatorio ampliando el plazo 15 días más.

\section{Análisis estadístico}

Para el análisis de los datos se empleó el paquete estadístico SPSS para Windows (versión 17.0).

Se realizan análisis descriptivos a través de medias y frecuencias. Por otro lado se estudian las relaciones entre variables, a través de Rho de Spearman. Finalmente se realiza un contraste de medias utilizando $t$ de Student para las variables cuantitativas. Las preguntas abiertas son categorizadas a través de frecuencias.

\section{RESULTADOS}

\section{Descripción de la muestra}

La muestra final es de 41 rotantes. Las rotaciones presentan un rango desde 15 
días a dos años. En esta muestra se incluye al coordinador del equipo de psicólogos. Un 7,3\% de los participantes ( $N=2)$ trabaja actualmente en otros servicios del centro hospitalario aunque participan semanalmente de esta dinámica grupal.

La media de edad fue de 32 años (D.T= $7,07)$ con un porcentaje de hombres de $11,4 \%$ y $88,6 \%$ de mujeres. En su mayoría los participantes son psicólogos (90,9\%), aunque también han participado médicos $(9,4 \%)$.

Las rotaciones en el servicio se realizan por estudiantes de posgrado $(72,7 \%)$ y por alumnos de último año de carrera o de grado $(27,3 \%)$.

\section{Análisis descriptivo de las diez variables del instrumento}

Todas las dimensiones a evaluar son altamente valoradas por los sujetos, mayor de 7 en escala tipo likert de 0 a 10 (Media Total $=8,32$ y D.T=11,68). Tan solo la variable identificación y resolución de conflictos, es valorada discretamente inferior, con una media de 6,36 y D.T=.2, 57. (Tabla 1).
Las variables más apreciadas han resultado ser Apoyo Mutuo, Sentido de Pertenencia, Expresión Emocional, Identificación Emocional y las aportaciones al Ámbito Personal. Los detalles de las variables evaluadas se recogen en la tabla 1.

\section{Relaciones entre las variables}

Se detecta una alta correlación entre Identificación y expresión emocional, entre Expresión emocional y Apoyo Mutuo y entre Apoyo Mutuo y Pertenencia. Ver la tabla 2.

\section{Contrastes entre grupos}

a) Trabajo actual con pacientes, dividido entre los que tras su rotación han trabajado como psicólogos o médicos en contacto con pacientes y los que no han tenido la oportunidad. Se aprecian diferencias significativas entre estar trabajando con pacientes actualmente y la valoración de la dinámica para la Adquisición de Herramientas clínicas y la Identificación de Emociones.

\section{Tabla 1. Estadístico descriptivo para 10 variables de la dinámica "¿Qué Tal?"}

\begin{tabular}{lcc}
\multicolumn{1}{c}{ Variable } & M & DT \\
\hline Autorregulación & 7,83 & 1,51 \\
Identificación y resolución de conflictos & 6,63 & 2,57 \\
Identificación emocional & 8,37 & 1,60 \\
Expresión emocional & 8,39 & 1,80 \\
Seguridad Profesional & 7,58 & 2,07 \\
Apoyo Mutuo & 8,80 & 1,34 \\
Pertenencia & 8,65 & 1,46 \\
Habilidades Sociales & 7,83 & 1,73 \\
Adquisición Herramientas Clínicas & 7,85 & 1,79 \\
Ámbito Personal & 8,17 & 1,69 \\
\hline
\end{tabular}




\section{Tabla 2. Correlaciones entre las variables de la dinámica “Qué Tal?"}

\begin{tabular}{lcccccccccc}
\multicolumn{1}{c}{ Variable } & $\mathbf{1}$ & $\mathbf{2}$ & $\mathbf{3}$ & $\mathbf{4}$ & $\mathbf{5}$ & $\mathbf{6}$ & $\mathbf{7}$ & $\mathbf{8}$ & $\mathbf{9}$ & $\mathbf{1 0}$ \\
\hline 1. Autorregulación & 1 & $0,49^{* *}$ & $0,431^{* *}$ & $0,47^{* *}$ & 0,198 & $0,343^{*}$ & 0,265 & 0,243 & 0,27 & $0,35^{*}$ \\
2. Conflictos & 1 & 0,23 & 0,127 & 0,198 & 0,143 & 0,129 & 0,111 & $0,40^{* *}$ & 0,167 \\
3. Id. emocional & & 1 & $0,7 * *$ & $0,453^{* *}$ & $0,569^{* *}$ & $0,521^{* *}$ & $0,520^{* *}$ & $0,573^{* *}$ & $0,61^{* *}$ \\
4. Ex. emocional & & & 1 & $0,46^{* *}$ & $0,69^{* *}$ & $0,59^{* *}$ & $0,46^{* *}$ & $0,36^{*}$ & $0,67^{* *}$ \\
5. Seguridad Prof. & & & & 1 & $0,47^{* *}$ & $0,38^{* *}$ & $0,36^{*}$ & $0,61^{* *}$ & $0,40^{*}$ \\
6. Apoyo Mutuo & & & & & 1 & $0,68^{* *}$ & $0,35^{*}$ & $0,34^{*}$ & $0,68^{* *}$ \\
7. Pertenencia & & & & & & 1 & 0,29 & $0,32^{*}$ & $0,59^{* *}$ \\
8. H.H. S.S. & & & & & & & 1 & $0,41^{* *}$ & $0,45^{* *}$ \\
9. Herramientas & & & & & & & & 1 & $0,39^{*}$ \\
10. Ám. personal & & & & & & & & & 1 \\
\hline
\end{tabular}

Nota: *Todos los coeficientes son significativos con una $\mathrm{p}<0,05$.

** Todos los coeficientes son significativos con una $p<0,001$.

\section{Tabla 3. Diferencias entre profesionales que trabajan actualmente con pacientes y los profesionales que no trabajan actualmente con paciente}

Trabajo actual con pacientes Sin contacto con pacientes

$\mathrm{N}=34$

$\mathrm{N}=7$

\begin{tabular}{lccccc}
\multicolumn{1}{c}{ Variables } & $\mathbf{M}$ & $\mathbf{D E}$ & $\mathbf{M}$ & $\mathbf{D E}$ & $\mathbf{T}$ \\
\hline Identificación & 8,68 & 1,429 & 6,86 & 2,340 & $0,005^{*}$ \\
Emocional & 8,85 & 1,149 & 7,71 & 2,360 & 0,061 \\
Pertenencia & 8,18 & 1,64 & 6,29 & 1,70 & $0,026^{*}$ \\
$\begin{array}{l}\text { Adquisición de } \\
\text { Herramientas }\end{array}$ & &
\end{tabular}

$* p<0,05$

b) Tiempo transcurrido desde la rotación en el equipo, dividido entre los participaron hace menos de cinco años y los que participaron hace cinco años o más. Los que tienen la experiencia más próxima, valoran significativamente mejor el aporte del "¿Qué-tal?" en la Expresión de las Emociones y al Ámbito de lo Personal. $(p<0,01)$. Ver Tabla 4.

Además de las apreciaciones anteriormente descritas, se realizaron dos comparaciones adicionales. En una se comparó a los participantes que rotaron durante menos de 12 meses y los que rotaron durante más tiempo y en la otra comparamos a los pacientes que ya disponían de la licenciatura o grado durante su rotación con los que se encontraban en el último año de formación. En ningún caso se encontraron diferencias significativas.

\section{Análisis Cualitativo}

Preguntas abiertas sobre la vivencia personal con la dinámica

La primera reunión con el "¿Qué-tal?" es recordada por prácticamente la totalidad de los participantes. En gran medida ese 


\section{Tabla 4. Diferencias entre profesionales que rotaron por el equipo hace menos} de cinco años y profesionales que rotaron por el equipo hace cinco años o más

\begin{tabular}{|c|c|c|c|c|c|}
\hline \multirow[b]{2}{*}{ Variables } & \multicolumn{2}{|c|}{$\begin{array}{l}\text { Rotación hace menos de } 5 \text { años } \\
\qquad N=24\end{array}$} & \multicolumn{3}{|c|}{$\begin{array}{l}\text { Rotación hace } 5 \text { años o más } \\
\qquad N=14\end{array}$} \\
\hline & $M$ & DE & $M$ & $\mathrm{DE}$ & $\mathbf{T}$ \\
\hline Expresión Emocional & 9,04 & 1,197 & 7,47 & 2,066 & $0,005^{*}$ \\
\hline Ámbito personal & 8,63 & 1,439 & 7,57 & 1,604 & 0,053 \\
\hline
\end{tabular}

$* \mathrm{p}<0,05$.

primer encuentro provocó incertidumbre en los rotantes aunque también sensaciones grupales como pertenencia y apoyo.

La mayor parte de los participantes consideran que la dinámica ha resultado útil para la gestión del mundo emocional, tanto a nivel grupal como personal.

El rasgo más destacable del uso de la dinámica tiene que ver con el apoyo mutuo percibido por el equipo. La mayoría no añadiría nada a este espacio.

Un porcentaje apreciable de participantes $(41,4 \%)$ asegura haber encontrado una herramienta similar en su ubicación laboral actual aunque menos sistemática.

Los que han intentado trasladar el "¿Qué-tal?" a su trabajo actual comentan resultados satisfactorios.

\section{Texto libre sobre la aportación de la herramienta para cada variable}

Los participantes consideran que la práctica de la dinámica "¿Qué-tal?" ha resultado beneficiosa para la adquisición de numerosas habilidades de carácter profesional y personal.

\section{DISCUSIÓN}

La presente investigación tiene por objetivo evaluar la utilidad percibida por los usuarios de la dinámica "¿Qué-tal?" como herramienta de autocuidado y aprendizaje mutuo. Los resultados indican una alta adecuación de la herramienta tanto a nivel profesional como personal.

El estudio cuenta con una alta tasa de respuesta $(82 \%)$. Esta amplia colaboración podría reflejar la satisfacción de los participantes con la dinámica.

La dinámica comprende la aportación de una serie de características relevantes en el desempeño asistencial. La valoración que los profesionales han realizado de cada una de estas características ha sido elevada (por encima de 7 en una escala de 0 a 10) evidenciando la utilidad de la herramienta en este campo.

Las aportaciones que han resultado más robustas se refieren al ámbito grupal (como la pertenencia y el apoyo mutuo), a la gestión emocional y al ámbito personal. En lo referente al sentimiento grupal, en concreto el sentido de pertenencia, puede deberse a que estos profesionales que forman equipo comparten una serie de objetivos y procedimientos. Para PichónRiviere $^{(14)}$ para estos equipos, la tarea resultará el eje de su condición grupal. El propio hecho de compartir, puede facilitar la expresión de las propias emociones ya que el grupo acoge nuestros miedos e inquietudes, se aprecia la idea de equipo como grupo que Hidalgo y Aylwin ${ }^{(15)}$ identifican como red de apoyo. Favorecer el 
entrenamiento de estas habilidades útiles en el terreno profesional, repercute en el ámbito personal, ya que todas las características que trabaja la herramienta son parte de la propia vida. Puede identificarse con la conclusión de Gil Monte y Peiró(16), donde se establece el reconocimiento del apoyo social como fuente de realización personal, factor que permite disminuir el agotamiento emocional y mejorar las actitudes hacia los demás.

Al estudiar las variables de la dinámi$\mathrm{ca}$, se encuentran correlaciones robustas entre las referidas a la gestión emocional: identificación y expresión emocional. Podríamos extraer de este hecho que a través de la dinámica, los participantes se familiarizan con la expresión de sus emociones y las de otros. La adquisición de esta habilidad favorece la identificación de las mismas. Podríamos decir que expresión e identificación emocional son dos variables que se potencian mutuamente.

Otras correlaciones de interés son las encontradas entre las variables relacionadas con el sentimiento de equipo: como son la percepción de apoyo mutuo y la de pertenencia. La pertenencia se experimenta en función del apoyo percibido. Sentirse perteneciente se da ante un reforzamiento mutuo, al ser escuchado, al participar de manera activa en los aspectos tratados, que son de interés común. En cambio saberse perteneciente, es tan solo formar parte del equipo, de forma pasiva. Podríamos vincular la experiencia de apoyo mutuo como una estrategia de pertenencia porque uno se siente perteneciente al tener una experiencia grupal conjunta, como lo es el "¿Qué-tal?". Para Morales $^{(17)}$, las estrategias de equipo están estrechamente relacionadas con los procesos grupales, entendiendo al grupo como un suprasujeto de un sistema.

Estos dos grupos de variables: gestión emocional y sentimiento de equipo, además correlacionan alto entre sí. Una po- sible explicación sería que todos necesitamos pertenecer a determinados grupos para usarlos de marcos de referencia en cuanto a lo válido en la gestión de las emociones. Este marco resulta más importante en los que trabajan en contacto con personas que sufren y expresan sus emociones. Para el grupo son importantes las diferencias individuales de sus miembros, lo que puede enriquecer al propio desempeño del equipo.

En el ámbito personal, existen correlaciones robustas para prácticamente todas las variables. Las más señaladas son las correlaciones entre la gestión emocional y la pertenencia. Todo lo que se trata en el "¿Qué-tal?" es importante a nivel personal. Se podría decir que se potencian habilidades profesionales generalizables al ámbito personal de forma automática. Del mismo modo, al "¿Qué-tal?" se aporta información personal dado que afecta al terreno profesional.

Al realizar las comparaciones entre perfiles del grupo, resulta interesante mencionar que no se aprecian diferencias en cuanto al tiempo dedicado a la rotación ni al nivel de estudios durante la misma. En cambio, los que tras su rotación se han tenido la oportunidad de volver a trabajar al lado de personas que sufren, valoran especialmente el "¿Qué-tal?" como buena estrategia para la adquisición de herramientas de carácter clínico y la identificación de emociones. Es posible que este grupo de profesionales no dispongan en su ubicación laboral actual de un equipo donde volcar sus inquietudes. Esta hipótesis cobra fuerza al comprobar que este grupo valora más la pertenecía grupal.

Otra diferencia que encontramos al comparar grupos es que los que rotaron por el servicio más recientemente, valoran más las aportaciones de la herramienta en la expresión emocional y en el ámbito personal. Probablemente esto suceda porque se tiene más conciencia de la repercusión 
en lo personal cuando existe más cercanía en el tiempo.

En la valoración cualitativa de la herramienta podemos llegar a algunas ideas claves, como que la primera reunión del "¿Qué-tal?" es recordada por la mayoría de los rotantes. Inicialmente provocando una alta sensación de incertidumbre que después daría paso a la gratificante sensación de acogida.

\section{LIMITACIONES}

Este estudio está realizado con una sola medida, ausencia de grupo control y cuenta con una $N$ pequeña. No se han empleado instrumentos estandarizados en la evaluación.

\section{CONCLUSIONES}

Tras el presente estudio podemos concluir que la estrategia "¿Qué-tal?" es una herramienta de autocuidado para equipos en contacto con el sufrimiento humano, valorada satisfactoriamente por sus usuarios, tanto a nivel profesional como personal.

Las principales aportaciones de esta dinámica grupal parecen radicar en las siguientes cuestiones:

- Parece imprescindible para el desarrollo profesional y personal en este tipo de profesionales, disponer de un tiempo y espacio explícito en la dinámica grupal para hablar de lo importante (aunque no sea urgente). Algo complicado de conseguir en este ámbito por la presión asistencial diaria.

- Es importante para todos apoyar y sentirse apoyados por los compañeros del equipo en todo lo relativo a la gestión de las emociones. De este modo convertimos al equipo asistencial en un potencial grupo de apoyo mutuo, con la consiguiente importancia en la prevención del Burnout.
- Se potencia la identificación y visualización de los problemas.

- Se proporciona el modelado de determinadas estrategias para detectar las emociones, ponerles nombre, compartirlas y apoderarse de ellas.

- Se facilita la posibilidad de dimensionar las emociones en el arco que va desde la normalidad a la sobreimplicación evitable, a través de una correcta canalización del mundo emocional.

- Se facilita un conocimiento más adecuado de las capacidades y potencialidades de cada compañero respetando la igualdad de exposición de experiencias personales.

- Permite profundizar en temas de interés que se perciban como recurrentes semanalmente o como altamente amenazantes para el equipo.

- Facilita la autoafirmación de forma crítica y constructiva del trabajo realizado.

Este estudio aporta la visión percibida por los participantes de la dinámica como muy positiva. Toda la información extraída parece determinar que la implantación de esta dinámica a grupos de trabajo asistenciales con alta implicación emocional, tendría resultados similares en satisfacción. Sin embargo el estudio actual no cuenta con un grupo control con el que comparar los resultados.

Se proponen líneas de investigación futuras en las que se desarrollen nuevas formas de intervención que, a partir de un modelo salutogénico, fomenten programas de intervención alternativos y flexibles.

De este modo se podrá tener en consideración la enorme variedad de reacciones que desencadena una misma situación traumática entre las diferentes personas expuestas. Esto es importante ya que sabemos que aquello que resulta traumático para uno, no lo es necesariamente para los otros. Las emociones suscitadas por el mismo evento no son iguales en todas las personas. 
Observamos que el "¿Qué-tal?" favorece la gestión del mundo emocional, que engloba tanto la regulación de las emociones a nivel grupal como personal. Resulta evidente que la dinámica aporta aprendizaje en el plano profesional, como la adquisición de herramientas de carácter clínico, la comunicación asertiva, el moldeamiento, etc.

AGRADECIMIENTOS: El equipo de psicólogos del Servicio de Hematología quiere expresar el agradecimiento a la COlaboración de todos los profesionales implicados en la realización de este estudio durante los años 2012 y 2013. Igualmente, se extiende el agradecimiento a todos los rotantes del equipo a lo largo de los seis años que analiza este estudio. Queremos especialmente dar las gracias a la Dra. Pilar Arranz, Laura Díaz Sayas y Erika Guijarro.

\section{REFERENCIAS BIBLIOGRÁFICAS}

1. Zapf D. Emotion work and psychological well-being: A review of the literature and some conceptual considerations. Hum Resour Manage R 2002;12:237-68. Doi: 10.1016/S1053-4822(02)00048-7

2. Figley CR. Compassion fatigue: Coping with secondary traumatic stress disorder in those who treat the traumatized. New York: Brunner/Mazelpublishers, 1995.

3. Aguilar MI. Trabajo interdisciplinario y cuidado de equipos. Seminario Salud mental y género, nuevos énfasis en las estrategias de trabajo, Servicio Nacional de la Mujer, Santiago, Chile, 1996

4. Moran C. Humor as a moderator of compassion fatigue. En: Figley $\mathrm{CR}$, editor. Treating compassion fatigue. New York: Brunner-Routledge, 2002.p.139-154

5. Barudy J. Maltrato infantil, ecología social: prevención y reparación. Barcelona: Paidós, 2000
6. Gentry JE. Desgaste por empatía: el desafío de la transformación. Revista de Psicotrauma para Iberoamérica 2003;2:417

7. Dyregrov A.. Helpful and hurtful aspects of psychological debriefing groups.

International. Int J Emerg Ment Health 1999;1:175-81.

8. Benson J, Magraith K. Compassion fatigue and burnout: The role of Balint groups. Aust Fam Physician 2005;34:497-8

9. Frankl V. El hombre en busca de sentido. Conceptos básicos de logoterapia. Barcelona: Herder, 1979

10. Lakoff, G., Narotzky, S., Millán, J.A., Johnson, M. Metáforas de la vida cotidiana. Madrid: Cátedra, 1986.

11. Pennebaker J.W. Writing about emotional experiences as a therapeutic process. Psychol Sci 1997;8:162-6 Doi: 10.1111/ j.1467-9280.1997.tb00403.x

12. Bayés R. Afrontando la vida, esperando la muerte. Madrid, Alianza Editorial. Cap.5, enfermedad y sufrimiento, 2006

13. Yalom I. Psicoterapia existencial y terapia de grupo. Barcelona, Paidós cap.4 las cuatro preocupaciones supremas, 1998.

14. Morales G. El equipo de intervención psicosocial como un grupo de alto riesgo. Salud y cambio. Revista Chilena de Medicina Social 1996;22:38-45

15. Hidalgo C, Aylwin N. Familia e interdisciplina. Documento de Trabajo 36. Programa de Postítulo de Estudios de la Familia. Santiago: Pontificia Universidad Católica de Chile \& Corporación de Promoción Universitaria, 1992

16. Peiró JM, Gil-Monte GM. Perspectivas teóricas y modelos interpretativos para el estudio del síndrome de quemarse por el trabajo. An Psicol 1999;15:261-8.

17. Morales G. El equipo de intervención psicosocial como un grupo de alto riesgo. Salud y cambio. Revista Chilena de Medicina Social 1996;22:38-45 


\section{Anexo 1. Cuestionario on line}

Estimado compañero, tal y como te hemos explicado en el mail que has recibido anteriormente, a continuación te presentamos una serie de preguntas acerca de la reunión de los viernes del equipo de psicólogos del Servicio de Hematología del Hospital Universitario La Paz, en la que - resumiendo- hablamos de nosotros y de cómo nos ha ido la semana. Experiencia que se ha denominado dinámica del "¿Qué-ttal?". El documento que tienes delante de ti está compuesto de tres partes. - Primera parte: breves datos sociodemográficos. - Segunda parte: ocho preguntas abiertas que intentan recoger tu vivencia personal con la dinámica del "¿Qué-Tal?". - Tercera parte: once preguntas cerradas tipo Likert (0-10), que pretenden evaluar la aportación que tuvo para ti la dinámica del "¿Qué-Tal?" como herramienta de autocuidado y aprendizaje mutuo. Te rogamos que respondas a las tres partes en su totalidad para poder favorecer la investigación. Muchas gracias por tu colaboración y por tu tiempo.

\section{Parte I: Datos Sociodemográficos}

Edad

Sexo

Profesión

Año en que comenzó la rotación

Tiempo aproximado de rotación

Formación durante la rotación

Trabajo actual con pacientes

Años posteriores de trabajo profesional

\section{Parte II: Preguntas Abiertas}

1. ¿Recuerdas el primer "Qué-Tal" de los viernes que hiciste? ¿Qué sensaciones destacarías?

2. ¿En qué te ha resultado útil el haber participado en la dinámica del "Qué

3. ¿Qué es lo que más destacarías de esta experiencia?

4. ¿Qué es lo que añadirías en esa dinámica de los viernes?

5. ¿Qué quitarías en la dinámica del "Qué-Tal"?

6. Si tuvieras que elegir una imagen (símbolo, escenario, sensación, color, música, etc) que exprese lo que para ti fue el "Qué-Tal", ¿Cuál elegirías?

7. ¿Has encontrado alguna herramienta similar a la dinámica del "Qué-Tal" en tu experiencia profesional posterior? ¿En qué consistía o consiste?

8. ¿Has intentado trasladar esta herramienta a otros espacios profesionales? ¿Con qué variantes y resultados?

\section{Parte III: Preguntas Cerradas}

Puntúa de 0 a 10 en qué te ha ayudado el "Qué tal?" para las siguientes variables y razona la respuesta.

1. AUTORREGULACIÓN

2. IDENTIFICACIÓN Y/O RESOLUCIÓN DE CONFLICTOS EN EL EQUIPO

3. IDENTIFICACIÓN DE EMOCIONES

4. EXPRESIÓN EMOCIONAL

5. ADQUISICIÓN DE SEGURIDAD PROFESIONAL

6. PERCEPCIÓN DE APOYO MUTUO

7. AUMENTO DE SENTIDO DE PERTENENCIA AL EQUIPO

8. MEJORA Y MODELADO DE HABILIDADES SOCIALES

9. ADQUISICIÓN DE HERRAMIENTAS DE MANEJO CLÍNICO

10. ÁMBITO DE LO PERSONAL 
